\title{
Correlación entre la estructura de bandas y las propiedades físicas de óxidos cerámicos de estructura perovskita con metales de transición (I): Propiedades de conducción electrónica.
}

\author{
L. SÁNCHEZ, J.R. JURADO \\ Instituto de Cerámica y Vidrio, CSIC, 28500 Arganda del Rey, Madrid.
}

\begin{abstract}
En este artículo se revisa los principales modelos teóricos, que pretenden dar una explicación sobre las propiedades de conducción electrónica de óxidos cerámicos con estructura perovskita, que incluyen en la posición B metales de transición del primer período $(\mathrm{Fe}, \mathrm{Co}, \mathrm{Ni}, \mathrm{Mn}$ ), en conexión con su estructura de bandas y el carácter de sus electrones más externos, así como las transiciones metal aislante. Una continuación de este trabajo (Parte II), tratará lo referente a propiedades magnéticas y electrocatalíticas intentando integrar los trabajos llevados a cabo en la literatura sobre los campos citados.
\end{abstract}

Palabras clave: óxidos cerámicos de tipo perovsquita, conducción electrónica, estructura de bandas, transiciones metal aislante.

Band structure and physical properties relationship in transition metal ceramic oxides with perovskite structure (I): Electronic conduction properties

Theoretical models present in the literature and developed for the explanation of electronic conduction properties in transition metal $(\mathrm{Fe}, \mathrm{Co}, \mathrm{Ni}, \mathrm{Mn})$ ceramic oxides with perovskite structure in relation with their band diagram and outer electron nature, as well as metal insulator transitions have been revised. The second part of this work will be involved with magnetic and electrocatalytic properties.

Key words: ceramic oxides with perovskite structure, electronic conduction, band structure, metal insulator transitions

\section{INTRODUCCIÓN}

El objeto de este trabajo es el estudio y revisión de los modelos planteados en la literatura sobre las propiedades de conducción electrónica, que caracterizan a los óxidos cerámicos tipo perovskita, que contienen metales de transición, y que se relacionan con la estructura de bandas, y el carácter de los electrones más externos del cristal. Su descripción puede basarse en la teoría de campo cristalino (átomos localizados en posiciones atómicas discretas, ya que las interacciones entre vecinos son débiles), o la de bandas (interacciones fuertes y electrones compartidos entre todos los núcleos). Para ello es fundamental comprender los criterios de aplicación de una $\mathrm{u}$ otra, porque se diferencian notablemente en procesos físicos, como fenómenos de transporte, propiedades magnéticas, propiedades ópticas y electrocatalíticas. Una visión general, (como la que se dará en el presente trabajo y su continuación), de todos estos aspectos, se hace necesaria para comprender de forma global el comportamiento de las distintas composiciones en función de la estructura de bandas que presentan. Se hace especial hincapié al hecho que, a lo largo de estos artículos de revisión, se incorporan de forma especial y frecuente, las excelentes aportaciones, que en este campo, ha realizado, y realiza el Profesor Goodenough.

La versatilidad composicional de la estructura perovskita resulta ideal para desarrollar un estudio sistemático y global como el propuesto, siendo además, la familia de óxidos considerada muy interesante a nivel conceptual y de aplicación industrial. Existe la posibilidad de una teoría simplificadora e integradora de todas las propiedades de estos compuestos y podría estar en conexión con la estructura de bandas.

\section{ESTRUCTURA DE BANDAS}

Se presentan algunos conceptos básicos de los modelos de electrones localizados, colectivos y de banda estrecha, así como un criterio de empleo del adecuado en cada caso. En general los electrones s y p son los principales responsables de la energía de enlace en el cristal, interaccionan fuertemente con los núcleos y son localizados mientras que los electrones 4f o $5 f$ enlazan débilmente con los núcleos y están apantallados frente a los vecinos, y tienen carácter colectivo. Los electrones d muestran un carácter intermedio con propiedades de localizados, colectivos o ambos según el tipo de cristal. En los óxidos propuestos, debido a las diferentes electronegatividades del catión y del anión es el carácter de los electrones d el que condiciona sus propiedades físicas.

\section{1 -Electrones colectivos (deslocalizados) y localizados.}

\subsubsection{ELECTRONES COLECTIVOS. HIPÓTESIS DE HARTREE-FOCK.}

En un sistema de electrones deslocalizados es necesario aplicar la hipótesis de Hartree-Fock $(1,2)$ según la cual, la función de onda (f.d.o) $\Psi$ de un sistema de $\mathrm{N}$ electrones itinerantes puede representarse por una función en forma de determinante de Slater cuyos elementos son funciones de onda de un electrón $\Phi\left(\tau_{\mathrm{j}}\right.$ ), conteniendo en $\tau_{\mathrm{j}}$ las tres variables espaciales y la de spin. El Hamiltoniano para este sistema de N electrones será:

$$
\mathrm{H}=\sum_{\mathrm{i}=1, \mathrm{~N}}\left[\left(\mathrm{p}_{\mathrm{i}}^{2} / 2 \mathrm{~m}\right)-\mathrm{V}_{\mathrm{i}}\right]+{ }_{-} \sum_{\mathrm{i}, \mathrm{j}=1, \mathrm{~N}} \mathrm{e}^{2} / \mathrm{r}_{\mathrm{i}, \mathrm{j}}
$$


El primer término es el operador de energía cinética (siendo $\mathrm{p}$ el momento y $\mathrm{m}$ la masa) y $\mathrm{V}_{\mathrm{i}}$ un potencial idéntico para todos los $\mathrm{N}$ electrones más externos. El último término representa la suma de las energías interelectrónicas de carácter coulombiano, siendo $r$ la distancia entre los electrones. Gracias a dicha hipótesis puede resolverse el problema separando (1) en $\mathrm{N}$ ecuaciones que darán como solución las funciones de onda para cada electrón es decir:

$$
H \Phi_{i}(\tau)=\varepsilon_{i} \Phi_{i}(\tau)
$$

donde $H=\mathrm{p}^{2} / 2 \mathrm{~m}+U(\tau)$

y la energía potencial se describe como:

$$
U=\mathrm{V}_{\mathrm{i}}+\mathrm{A}-\mathrm{C}
$$

Tanto A como C son energías coulombianas, la primera depende del spin de los electrones y es la energía de canje mientras que la segunda es independiente del spin. La energía total del sistema será la suma de las energías de todos los estados ocupados.

La superficie de Fermi (1) que aparece en el espacio de momentos es una característica fundamental de los estados electrónicos colectivos, cuando las energías de interacción entre electrones son suficientemente altas como para eliminar su sentido físico los electrones que ocupan distinta posición atómica pasan a ser distinguibles y se consideran localizados. En un cristal la carga positiva se localiza generando un potencial periódico de la forma:

$$
\mathrm{V}_{\mathrm{i}}=\sum_{\mathrm{v}=1, \mathrm{~N}} \mathrm{Z}_{\mathrm{v}} \mathrm{e}^{2} /\left|\mathbf{r}-\mathbf{R}_{\mathrm{v}}\right|
$$

Se tiene en cuenta la carga efectiva del núcleo del ión y sus capas electrónicas internas en la posición de la red es decir:

$$
\mathbf{R}_{\mathrm{v}}=\sum_{\mathrm{i}=1,2,3} v_{\mathrm{i}} \mathbf{a}
$$

Factorizando la parte espacial y de spin de la fdo de un electrón la primera será una función llamada de Bloch, de modo que el potencial completo, $U(\mathbf{r})$, presentará también la periodicidad de la red. Este es el fundamento de las teorías de electrones colectivos (deslocalizados) y de bandas. Las interacciones electrón-fonón (solamente se contemplan vibraciones térmicas de los defectos o de las capas electrónicas internas en la red) se tratan vía método perturbativo $(3,4)$.

\subsubsection{ELECTRONES CASI LOCALIZADOS. APROXIMACIÓN DE ENLACE FUERTE.}

Este modelo presenta al sólido como una colección de átomos interaccionando débilmente, y resuelve el caso en que las fdo atómicas solapan lo suficiente, como para requerir correcciones al esquema de átomos aislados, pero no tanto como para considerar, que la descripción atómica sea plenamente irrelevante. Es útil, fundamentalmente para las bandas procedentes de metales de transición, con niveles d parcialmente llenos, o para aislantes. La idea básica es que al disminuir la interacción entre los orbitales, las bandas se estrechan, disminuye la conductividad y finalmente se iría a estados localizados en el átomo $(1,2)$

Si las interacciones entre átomos vecinos son pequeñas, la parte espacial de la fdo se puede tomar como una función de
Bloch de la forma:

$$
\varphi_{\mathbf{k}}(\mathbf{r})=1 /(\mathrm{N})^{1 / 2} \sum_{\mathrm{n}=1, \mathrm{~N}} \exp \left(i \mathbf{k} \mathbf{R}_{\mathrm{n}}\right) \mathrm{w}\left(\mathbf{r}-\mathbf{R}_{\mathrm{n}}\right)
$$

donde $w\left(\mathbf{r}-\mathbf{R}_{\mathrm{n}}\right)$ es la fdo localizada para el átomo en $\mathbf{R}_{\mathrm{n}^{\prime}}$ en el caso límite de que el electrón esté enlazado al núcleo esas funciones son soluciones del hamiltoniano del campo cristalino. En general se toman como funciones de Wannier (1). En los óxidos perovskíticos las interacciones de los orbitales d de los cationes con los aniones introducen términos de hibridación covalente en estos orbitales de Wannier.

En esta aproximación se emplea la teoría de perturbaciones (2) para evaluar las energías del sistema para cada electrón:

$$
\varepsilon_{\mathrm{k}}=\left(\varphi_{\mathrm{k}^{\prime}} H \varphi_{\mathrm{k}}\right) /\left(\varphi_{\mathrm{k}^{\prime}} \varphi_{\mathrm{k}}\right)=\varepsilon_{0}+\varepsilon_{\mathrm{mn}}
$$

$\varepsilon_{0}$ es la energía del electrón en el campo cristalino sin tener en cuenta las interacciones, y la corrección es:

$$
\varepsilon_{\mathrm{mn}}=-\Sigma_{\mathrm{nm}} \mathrm{b}_{\mathrm{mn}} \exp \left(i \mathbf{k}\left(\mathbf{R}_{\mathrm{m}}-\mathbf{R}_{\mathrm{n}}\right)\right) / \mathrm{N}
$$

Donde el elemento $b_{m n}$ se denomina integral de transferencia o energía de transferencia $(1,4)$ y su valor es siempre positivo. Se trata de un indicativo de la fuerza de la interacción entre átomos vecinos similares, y se puede demostrar que su cuadrado es proporcional a la integral de solapamiento que veremos más adelante. El parámetro $\mathrm{b}$ permite una medida fenomenológica de la fuerza de interacción entre átomos, y también en el límite de enlace fuerte, las anchuras de las bandas de energías permitidas son proporcionales a él a través de $\varepsilon_{\mathrm{k}}$.

\subsubsection{ELECTRONES LOCALIZADOS. TEORÍA DE CAMPO CRISTALINO.}

El carácter localizado de los electrones d en algunos compuestos de los estudiados puede contemplarse a través de la ecuación de Schröedinger que describe esos electrones en sus posiciones atómicas discretas con el Hamiltoniano siguiente $(5,6,7)$ :

$$
H=H_{0}+V_{i n t}+V_{c r}+\left(V_{L S}+V_{\lambda}+H_{Z}+\Sigma_{j} V_{i j}\right)
$$

Siguiendo a Goodenough (2) podemos analizar cada uno de los términos presentes en la anterior expresión.

1. $H_{0}$ es la energía de un solo electrón en un potencial esférico, debido al núcleo al que está ligado, y al potencial medio del resto de los electrones, sus soluciones son funciones de onda del tipo de las del átomo de hidrógeno.

2. $V_{\text {int }}$ es una energía de canje intraatómica que corrige al modelo de Hartree-Fock cuando existe más de un electrón d por catión, de modo que aparece un desdoblamiento de los estados de diferente spin en una energía (llamada de canje) $\Delta_{\text {canje }} \approx \mathrm{S}(\mathrm{S}+1)$. Así el estado de menor energía mostrará el máximo valor de L y S. Este término no altera la dependencia angular de las fdo.

3. $V_{c r}$ es una perturbación que surge por el hecho de tratar con un cristal y contempla tanto la componente cúbica del campo cristalino como la no cúbica. La interacción entre estos potenciales cristalinos y los orbitales atómicos genera desdoblamientos de estos últimos.

Los orbitales atómicos pueden clasificarse según su orientación en un sistema de coordenadas cartesianas, en el caso particular de los $\mathrm{d}_{\text {tanto }} \mathrm{d}_{(\mathrm{x} 2-\mathrm{y} 2)}$ como $\mathrm{d}_{(\mathrm{z} 2)}$ están dirigidos en la 
dirección de los ejes principales mientras que $\mathrm{d}_{\mathrm{yz}}, \mathrm{d}_{\mathrm{zx}} \mathrm{y} \mathrm{d} \mathrm{d}_{\mathrm{xy}}$ lo están entre esos ejes. En un campo octaédrico como el que generan los aniones sobre la posición B en la perovskita, los electrones en los orbitales $\mathrm{d}_{(\mathrm{x} 2 \mathrm{-}-\mathrm{y} 2)} \mathrm{y} \mathrm{d}_{(\mathrm{z} 2)}$ sufren una repulsión electrostática mayor que los otros tres que se alejan de los aniones, por ello el nivel d se divide en un nivel doblemente degenerado menos estable $\mathrm{E}_{\mathrm{g}}\left(\mathrm{d}_{(\mathrm{x} 2-\mathrm{y} 2)}, \mathrm{d}_{(\mathrm{zz} 2)}\right)$, y otro triplemente degenerado $\mathrm{T}_{2 \mathrm{~g}}\left(\mathrm{~d}_{\mathrm{yz}}, \mathrm{d}_{\mathrm{zx}}, \mathrm{d}_{\mathrm{xy}}\right)$ de menor energía que el original, la separación energética entre ambos es $\Delta=10 \mathrm{Dq}(5,7)$ donde además de la contribución por interacciones electrostáticas la hibridación covalente tiene gran importancia. Se pueden sustituir las funciones de los orbitales atómicos (catiónicos y aniónicos) por otras funciones de campo cristalino $\varphi$ relacionadas con las primeras a través de las constantes de hibridación :

$$
\lambda \approx \mathrm{b}^{\mathrm{ca}} /\left(\mathrm{E}_{\mathrm{c}}-\mathrm{E}_{\mathrm{a}}\right)
$$

que son directamente proporcionales a la integral de transferencia $b^{\text {ca }}$ (catión-anión) de los orbitales que se mezclan e inversamente proporcionales a la energía que separa sus niveles.

Aparecen así de forma natural las denominadas integrales de solapamiento entre distintos orbitales cristalinos, que están directamente relacionadas con las energías de transferencia a través de esos $\lambda$, y cuya importancia radica en servir de indicativo de la intensidad de las interacciones y fuerza de enlace de los electrones con los núcleos. Son esas integrales las que permiten a Goodenough y col. $(2,5,7)$ definir un criterio de utilización del modelo de cargas localizadas o colectivas, así como distinguir propiedades de conducción y magnéticas y que pretendemos extender también al caso de las propiedades electrocatalíticas. A través de las expresiones de estas integrales de solapamiento (7) se deduce inmediatamente que son proporcionales al cuadrado de las integrales de transferencia. La notación empleada para identificarlas es la siguiente: $\Delta_{c c}$ (catión-catión), $\Delta_{\text {cac }}{ }^{\sigma}$ (catión-anión-catión, mediante enlaces $\sigma), \Delta_{\text {сас }}^{\pi \sigma}$ (idem, mediante un enlace $\pi$ y otro $\left.\sigma\right), \Delta_{\text {сас }}^{\pi}$ (idem, a través de enlaces $\pi$ ).

Cuando $\Delta_{\text {canie }}>10 \mathrm{Dq}$ el catión se encuentra en bajo estado de spin, mientras que para $\Delta_{\text {canie }}<10 \mathrm{Dq}$ estará en estado de alto spin. En óxidos de estructura perovskita sólo los iones divalentes o trivalentes del primer periodo presentan alto estado de spin, salvo el caso del Ni trivalente que está en estado de bajo spin y los cationes trivalentes de Co que muestran una población variable de alto o bajo spin en función de la temperatura.

4. $V_{L S}$ es el término relativo al acoplo spin-órbita.

5. $V_{\lambda}$ se refiere al acoplo elástico entre posiciones atómicas e implica interacciones cooperativas en el caso de las deformaciones cristalinas.

6. En $\Sigma_{j} V_{i j}$ el término preponderante es la energía de acoplo magnético en la aproximación de campo molecular y en las perovskitas contiene las interacciones spin-spin entre electrones d localizados en cationes de metales de transición vecinos.

7. $H_{Z}$ se refiere a la energía de Zeeman debida a ese campo interno generado por el orden magnético.

\subsection{La estructura perovskita y el $\mathrm{ReO}_{3}$}

La estructura perovskita ideal, $\mathrm{ABO}_{3}$, es cúbica con el grupo espacial Pm3m. (9). Los cationes B se sitúan en las ocho esquinas y los oxígenos en los puntos medios de las aristas generando la configuración $\mathrm{BO}_{3^{\prime}}$ de modo que la coordinación es de tipo octaédrico en las esquinas $\left(\mathrm{BO}_{6}\right)$. El catión A ocupa los centros de la celda cúbica y su índice de coordinación es 12 .

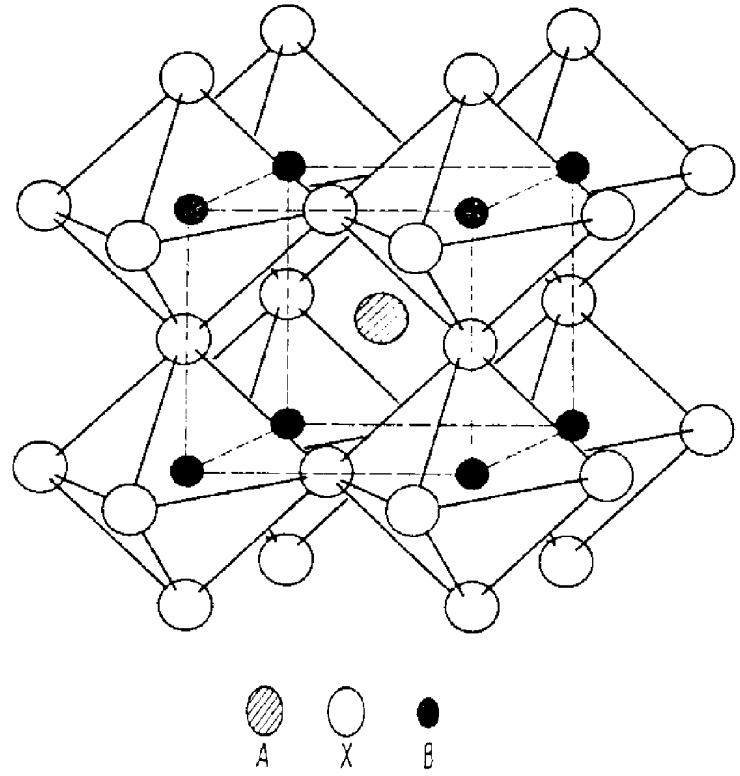

Fig 1. Estructura cristalina de la perovskita ideal $\mathrm{ABX}_{3}$.

A pesar de ser cúbicos a altas temperaturas, la mayor parte de estos compuestos $\mathrm{ABO}_{3}$ sufren deformaciones, que disminuyen la simetría por debajo de una cierta temperatura debido a los desplazamientos de las posiciones atómicas. El origen físico de estas transformaciones puede ser: a) la diferencia entre los tamaños iónicos, o b) la ordenación de electrones en estados localizados o colectivos $(7,9)$. a) En el primer caso basta con estudiar el factor de tolerancia definido por Goldschmidt (10):

$$
\mathrm{t}=\left(\mathrm{r}_{\mathrm{A}}+\mathrm{r}_{\mathrm{O}}\right) / \sqrt{ } 2\left(\mathrm{r}_{\mathrm{B}}+\mathrm{r}_{\mathrm{O}}\right)
$$

Donde $\mathrm{r}_{\mathrm{A}} \mathrm{r}_{\mathrm{B}}, \mathrm{r}_{\mathrm{O}}$ son los radios iónicos. Cuando $\mathrm{t}=1$ aparece la estructura ideal descrita, aunque también puede darse para $1<t<0.7$, sin embargo surgen otros condicionantes en este caso, ya que los iones A y B deben ser estables en sus coordinaciones 12 y 6 respectivamente; en los óxidos esto limita sus radios a $r_{A}>0.9$ y $r_{B}>0.51$ A. Para valores inferiores de $t$ se producen deformaciones de la estructura cúbica para optimizar las distancias de enlace A-O. Cuando $0.75<\mathrm{t}<0.9$ aparece una celda unitaria ortorrómbica debida las distorsiones de los octaedros $\mathrm{BO}_{6^{\prime}}$ para valores de $t$ ligeramente inferiores a la unidad aparece una estructura romboédrica (11). b) La segunda posibilidad es una transición a una fase de menor simetría debido a la localización de electrones d o por su presencia en una banda estrecha $(12,13)$. La localización induce cambios locales situando los cationes del metal de transición en los centros de simetría de los intersticios aniónicos. Los electrones de la banda modifican la simetría traslacional introduciendo planos de discontinuidad en la superficie de Fermi. Transiciones ferroeléctricas o semiconductor-metal ilustran deformaciones generadas por electrones colectivos. Además están las transiciones de Jahn Teller (5) que aparecen cuando el momento angular orbital varía por efecto d el campo cristalino de modo que el efecto del acoplo spin-órbita puede ser lo suficientemente intenso como para generar una deformación espontánea de la red que reduzca la energía eliminando la degeneración del estado fundamental. 
El $\mathrm{ReO}_{3}$ es una estructura importante para ilustrar el desarrollo de este trabajo por su alta conductividad y su parecido con la perovskita ideal, salvo por la ausencia del catión A. Podemos hacer un estudio detallado de los enlaces (14) que se dan en este compuesto y el diagrama de bandas propuesto por Goodenough $(2,7)$. En el caso del Re se produce una hibridación $\mathrm{sp}_{\sigma}^{3}$ y por otra parte en el oxígeno surge una hibridación $\mathrm{sp}_{\sigma}$. De este modo, se configuran cuatro enlaces sigma entre los orbitales hibridados $\mathrm{sp}_{\sigma}$ de cuatro oxígenos y el $\mathrm{sp}^{3}{ }_{\sigma}$ del Re, y otros dos enlaces sigma entre los dos orbitales e del Re y los $\mathrm{sp}_{\sigma}$ de otros dos oxígenos. Se genera así a lo largo del sólido una banda $\sigma$ llena y otra $\sigma^{*}$ vacía que se subdivide en la procedente del enlace con los orbitales $\mathrm{e}_{\mathrm{g}} \mathrm{y}$ con el $\mathrm{sp}^{3}{ }_{\sigma}$ del catión, en el caso del Re estas subbandas no solapan, pero puede suceder que sí lo hagan en otras composiciones. Por otra parte, aún restan tres orbitales $t_{2 g}$ que enlazan con los orbitales $\mathrm{p}_{\pi}$ de tres oxígenos generando una banda $\pi$ y otra $\pi^{*}$. Por último quedan tres orbitales $\mathrm{p}_{\pi}$ de tres oxígenos que apuntan unos a otros a lo largo de varias caras del cubo. Su separación es excesiva para que se dé un solapamiento suficiente,

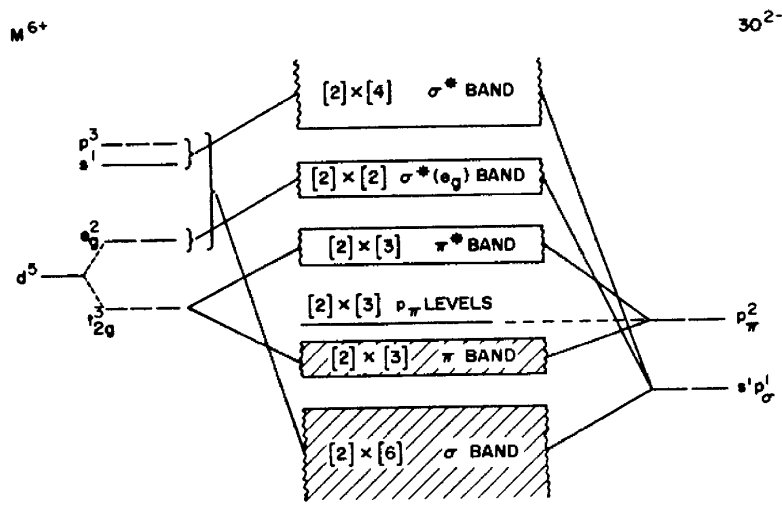

Fig 2. Esquema elemental de la estructura de bandas del $\mathrm{ReO}_{3}$ y sistemas a los que es extrapolable (Vest y Honing (14)). Se muestran los orbitales atómicos pertenecientes a los iones que luego dan lugar a la configuración de bandas del sistema así como su carácter y procedencia que aparece indicado entre paréntesis.

de modo que se trata de estados atómicos que no entran en el enlace apareciendo en el diagrama como estados localizados que pueden ser ocupados por 6 electrones.

Para determinar el nivel de Fermi bastaría con llenar estas bandas con los electrones disponibles. Si existe cualquier electrón de valencia en el catión $\mathrm{B}$ del sistema $\mathrm{BO}_{3}$ ocupará posiciones en las bandas por encima de los niveles $\mathrm{p}_{\pi^{\prime}}$ ya que los que están por debajo se llenan con los de los oxígenos. Este sistema tan simplista sólo sirve bajo las aproximaciones de enlace fuerte y la consideración de interacciones a primeros vecinos. Dado que el Re es un elemento pesado debería tenerse en cuenta los efectos de acoplo spin-órbita que aquí no se tratan. Estamos ante un modelo excesivamente básico y que no reproduce la curva de densidad de estados ni contempla las interacciones electrón-fonón y electrón-electrón salvo como perturbaciones pero aún así sirve de guía en un análisis de las cuestiones esenciales. Allí donde sea preciso, al tratar el modelo las correcciones al mismo se introducirán con detalle.

\subsubsection{PEROVSKITAS $\mathrm{ABO}_{3}$}

Los cationes A presentes en óxidos con la estructura perovskita tienen carácter básico y esencialmente se puede considerar que, en primera aproximación, no interaccionan lo suficiente con el anión $\mathrm{O}^{2-}$ ni con el catión B como para afectar a la estructura de bandas en torno al nivel de Fermi. De este modo su única función consistiría en donar electrones que se integrarían en ella y el diagrama anterior para el sistema $\mathrm{BO}_{3}$ sería extrapolable a las perovskitas. Sin embargo existen una serie de cuestiones a tener en cuenta y que Goodenough (2) clasifica como sigue:

a) Los cationes A compiten con los B por el enlace con los orbitales p del anión. Si se produce el enlace A-O no existe la banda $\pi^{*}$ prevista. Experimentalmente se ha observado que cuanto mayor sea el carácter ácido del catión A más estrecha es dicha banda.

b) Los cationes A además introducen cationes B de menor valencia (disminuye $\lambda_{\pi}$ ) y tienden a estrechar aún más la banda $\pi^{*}$.

c) La integral de solapamiento es mayor cuanto mayor sea la extensión radial de los orbitales d del catión B. Será muy superior para el $\operatorname{Re}(5 \mathrm{~d})$ que para los metales de transición con orbitales $3 \mathrm{~d}$, así puede que no se den los enlaces con el oxígeno que generan la formación de las bandas del modelo anterior.

d) Los cationes B pueden promoverse a un estado de alto spin como consecuencia de la interacción de canje, estrechando la banda $\sigma^{*}\left(\mathrm{e}_{\mathrm{g}}\right)$ y pudiendo aparecer electrones localizados en niveles atómicos $\mathrm{e}_{\mathrm{g}}$.

El modelo descrito, basado en la teoría de orbitales moleculares, nos permite contar con esquemas altamente simplistas pero que han demostrado ser aproximaciones razonables.

\section{CONDUCCIÓN ELECTRÓNICA. TRANSICIONES METAL-AISLANTE.}

\subsection{Propiedades de conducción de las perovskitas.}

Los óxidos cerámicos cubren todo el espectro de propiedades eléctricas $(14,15,16)$ : aislantes, superconductores, y conductores metálicos, (en este caso óxidos metálicos diferentes de los metales en la ionicidad, impurezas, en la concentración y en la distribución de electrones de conducción en el material). Según lo expuesto las perovskitas $\mathrm{ABO}_{3}$ con la configuración $\mathrm{d}^{1}-\mathrm{d}^{5}$ o d $\mathrm{d}^{7}-\mathrm{d}^{9}$ en $B$ presentarán una banda $\pi^{*}$ o $\sigma^{*}\left(\mathrm{e}_{\mathrm{g}}\right)$ parcialmente llena y con conducción metálica (figura 3).

Tomando como parámetro de referencia las integrales de solapamiento, Goodenough (7) establece un valor crítico, $\Delta_{\text {crit' }}$ por encima del cual se aplica la teoría de electrones colectivos y por debajo la de los localizados. Clasifica así los óxidos en tres grupos:

a) Clase 1 - son aquellos en que $\Delta_{\text {cc }}>\Delta_{\text {crit }}\left(\Delta_{\text {cac }}<\Delta_{\text {crit }}\right.$ para todos los orbitales d ocupados), por tanto tiene una banda de estados d en la subred de cationes y está parcialmente llena.

b) Clase $2 \mathrm{a}$ - cumplen que $\Delta_{\text {cac }}^{\sigma}>\Delta_{\text {crit }}$ (pero $\Delta_{\text {cc }}<\Delta_{\text {crit }}^{\prime}$ ), presentan banda estrecha y parcialmente llena.

c) Clase $2 \mathrm{~b}$ - en ellos $\Delta_{\text {cac }}^{\pi}>\Delta_{\text {crit }}$ (pero $\Delta_{\text {cc }}<\Delta_{\text {crit }}^{\prime}$ ), presentan banda estrecha y parcialmente llena.

También existen óxidos simultáneamente de clase 1 y 2 pero en el caso concreto de las perovskitas la separación entre los cationes del metal de transición es tan grande que siempre se cumple que $\Delta_{c c}<\Delta_{c \text { crit }}^{\prime}$ y sólo pueden pertenecer a la clase 2a o 


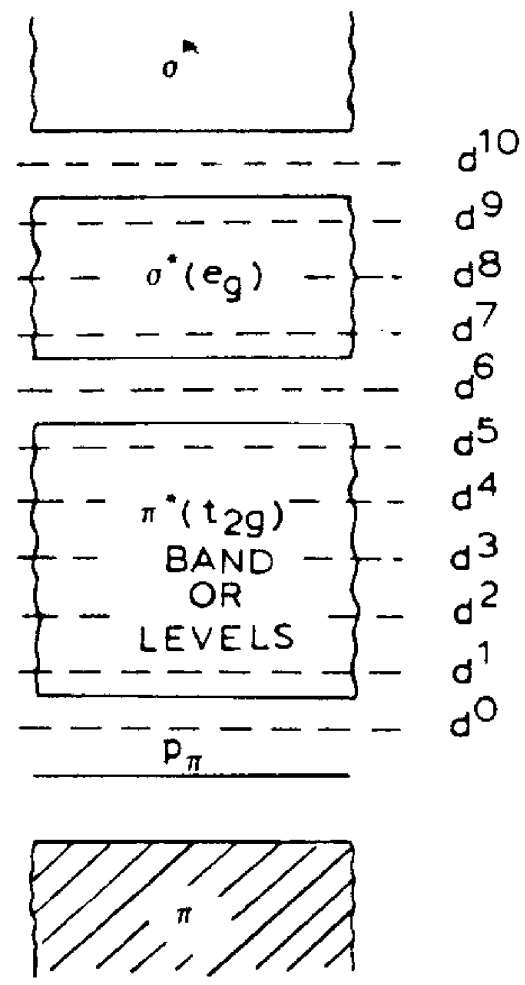

Fig 3- Niveles de Fermi para las distintas configuraciones electrónicas del catión $\mathrm{B}$ en los óxidos cerámicos $\mathrm{ABO}_{3}$. La banda de valencia aparece llena debido a los electrones del oxígeno.

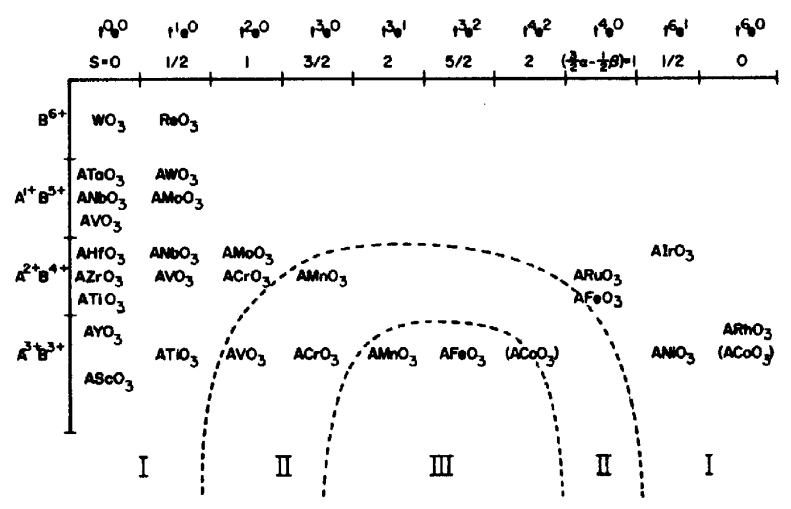

Fig 4. Regiones de conductividad de los óxidos tipo perovskita. Se representa el valor neto de spin frente a la configuración electrónica de B, según Goodenough (2)

2b. Por lo tanto el estudio del carácter de cada composición puede hacerse empleando como parámetro la $\Delta_{\text {cac }}$ de modo que cuando sea menor que cierto valor crítico hablaremos de

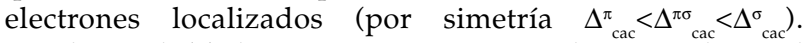
Goodenough (2) distingue tres regiones en las perovskitas, al representar el valor neto de spin frente a la configuración electrónica del óxido (figura 4).
En la región I el catión B presenta un estado de bajo spin y alta valencia, el solapamiento de los orbitales catión-anión es suficientemente extenso para que todos los electrones d sean itinerantes y se muestre comportamiento metálico siempre que exista una banda semillena. Ilustra el caso de una perovskita de clase $2 \mathrm{~b}\left(\mathrm{ReO}_{3}\right.$ y $\mathrm{AFeO}_{3}$ con $\left.\mathrm{Fe}^{4+}\right)$.

En la región II estados de spin y valencia intermedia se permite, que los efectos de canje sean lo suficientemente intensos, como para desdoblar los estados d en dos subredes de spin, separados en una energía $\Delta_{\text {canie }}$ que sean estados localizados o estén dentro de una banda. No se da la formación de la banda $\pi$ pero sí la de la $\sigma^{*}\left(\mathrm{e}_{\mathrm{g}}\right)$, así coexisten electrones localizados $\mathrm{t}_{2 \mathrm{~g}}$ y electrones itinerantes e en el caso de haberlos. Se trata de perovskitas de clase 2a. Los compuestos con catión B de configuración $\mathrm{d}^{7}-\mathrm{d}^{9}$ tendrán una banda $\sigma^{*}$ parcialmente llena y propiedades metálicas mientras que cualquier otra configuración generará propiedades semiconductoras aunque su conductividad sea alta ya que la energía de activación es pequeña $\left(\mathrm{AMnO}_{3}\right.$ con $\left.\mathrm{Mn}^{4+}\right)$.

La región III contiene materiales con el catión B en estado de alto spin y baja valencia. El solapamiento es tan pequeño que se localizan todos los electrones d. Así pues, en esta región sólo existen semiconductores o aislantes y las bandas $\pi^{*}$ y $\sigma^{*}$ son sustituidas por orbitales localizados $\mathrm{t}_{2 \mathrm{~g}}^{*} \mathrm{y} \mathrm{e}_{\mathrm{g}}^{*}$ respectivamente (ferratos, cobaltatos y manganitas con el catión de transición en estado 3+).

Goodenough $(2,7)$ establece también un criterio para los óxidos de metales de transición $3 \mathrm{~d}$ con estructura perovskita tomando cationes $\mathrm{B}$ de valencia $3^{+} \mathrm{o} 4^{+}$

Siguiendo estos criterios de clasificación, ampliamente aceptados y presentes en la literatura, como primera aproximación podremos predecir algunas de las propiedades básicas de conducción de compuestos como los propuestos. Sin embargo hay que señalar que para que un valor definido de la integral de solapamiento crítica exista, y para que el modelo tenga aplicación es preciso que estemos tratando con materiales en sus condiciones intrínsecas y que condiciones externas o manipulaciones ( $\mathrm{p}_{\mathrm{O} 2}$ dopado, no estequiometría...) pueden modificar los procesos de conducción $(15,17)$. Además existen ciertos comportamientos importantes que el modelo no contempla y correcciones necesarias a hacer como mostramos a continuación.

TABLA 1. ÓXIDOS DE METALES DE TRANSICIÓN Y ESTRUCTURA PEROVSKITA CON CONDUCCIÓN ELECTRÓNICA . ELABORACIÓN PROPIA CON DATOS RECOGIDOS DE TSUDA Y COL. (16) Y DE VEST Y HONING.(14)

\begin{tabular}{|c|c|}
\hline Compuesto & Resistividad específica a $300^{\circ} \mathrm{K}(\Omega \mathrm{cm})$ \\
\hline $\mathrm{ReO}_{3}$ & $110^{-7}$ \\
\hline $\mathrm{CaFeO}_{3}$ & $0.3, \mathrm{~d} \rho / \mathrm{dT}<0,<115^{\circ} \mathrm{K}$ \\
\hline $\mathrm{SrFeO}_{3}$ & $10^{-3}$ \\
\hline $\mathrm{SrCoO}_{3}$ & $10^{-4}$ (sinterizado) \\
\hline $\mathrm{YCoO}_{3}$ & Metálico por encima de $1200^{\circ} \mathrm{K}$ \\
\hline $\mathrm{ErCoO}_{3}$ & Metálico por encima de $1200^{\circ} \mathrm{K}$ \\
\hline $\mathrm{LaCoO}_{3}$ & $\begin{array}{l}\mathrm{d} \rho / \mathrm{dT}<0, \mathrm{~T}>700^{\circ} \mathrm{K} \\
\mathrm{d} \rho / \mathrm{dT}>0, \mathrm{~T}<600^{\circ} \mathrm{K} \\
\text { transición metal-aislante gradual entre } 520-720^{\circ} \mathrm{K}\end{array}$ \\
\hline $\mathrm{LaNiO}_{3}$ & $10^{-5}$ (sinterizado) \\
\hline $\mathrm{SrRuO}_{3}$ & $10^{-3}$ \\
\hline $\mathrm{CaRuO}_{3}$ & $10^{-3}$ \\
\hline
\end{tabular}


3.2 Correcciones al modelo: Conducción polarónica. Superconductividad. Conducción por saltos (hopping) variable.

El modelo de Hartree-Fock y la aproximación adiabática se muestran incapaces de describir algunos fenómenos de transporte que aparecen en estos óxidos al no tener en cuenta interacciones intensas entre electrones y entre ellos y los fonones (18). En general, la conducción electrónica en los óxidos cerámicos viene condicionado por tres elementos competitivos (16): el carácter colectivo de los electrones (a través de la integral de solapamiento $\Delta$, o de la de transferencia $\mathrm{b}$ ), la repulsión coulombiana entre electrones (a través de la diferencia en energía de un estado doblemente ocupado y otro en que únicamente se sitúa un electrón), y por último el acoplo electrón-fonón de modo que un electrón puede generar una deformación local de la red que disminuya el estado energético de un orbital y quedando atrapado en él. Dado que este nivel de energía reducida puede atrapar aún otro electrón la consecuencia global puede ser una atracción efectiva entre electrones.

\subsubsection{ACOPLAMIENTO ELECTRÓN-FONÓN. POLARONES.}

Las interacciones entre los electrones y los modos ópticos pueden tratarse a partir del modelo de Fröhlich $(1,19)$, que utiliza un sistema de cargas puntuales teniendo en cuenta la polarización atómica en el sólido. Los fonones longitudinales interaccionan intensamente con los electrones, mientras que los transversales lo hacen si su longitud de onda es pequeña. El polarón (20) es una partícula ficticia, que contempla esta interacción consistiendo en un electrón que se traslada junto a la polarización de la red que minimiza su energía. Cuando la deformación de la red se extiende a lo largo de un gran número de parámetros de red se genera un "gran polarón", si el acoplo es débil los niveles sólo se modifican reduciendo levemente su energía e incrementando ligeramente la masa efectiva, pero si es intenso puede suceder que la masa efectiva sea tan grande que el polarón difícilmente se mueva al aplicar un campo eléctrico. De todos modos la formación de estos grandes polarones no modifica sensiblemente las propiedades de transporte de la banda. Cuando la extensión de la deformación es pequeña comparada con la constante de red se habla de "pequeño polarón", y para un cristal perfecto, según Holstein (21), la superposición de estados de pequeño polarón es suficiente para constituir una banda polarónica (22) en la que la conducción sucede a bajas temperaturas ya que su anchura decrece exponencialmente con T. Por encima de $\theta_{\mathrm{D}} / 2$ el transporte en esta banda es despreciable y el pequeño polarón pasa a estar localizado siendo el único modo de poder contribuir a la conducción de este pequeño polarón el salto o "hopping" de una posición de red a otra equivalente, obligando a nuevas distorsiones, que precisan de una energía en la forma de modos ópticos longitudinales. Son procesos activados térmicamente, al igual que la conducción en la banda, y no pueden distinguirse sólo por medidas de conductividad eléctrica. Además el salto puede suceder siempre que existan estados localizados (caso de cargas situadas en donores o aceptores a bajas temperaturas). $\mathrm{Ni}$ en los metales, ni en los semiconductores con impurezas o defectos está presente la conducción polarónica. En el primer caso por la alta concentración de carga libre y en el segundo por las fluctuaciones en la energía entre posiciones vecinas debido a las impurezas aleatoriamente distribuidas.

\subsubsection{CONDUCCION POR SALTOS VARIABLE.}

Cuando el potencial periódico de la red es perturbado pueden aparecer estados localizados. Este proceso puede ser estático o dinámico, dependiendo del tipo de perturbación a que está sometido el potencial que excita al electrón. Según Mott (23), los bordes superior e inferior de las bandas son los que sufrirán esta localización de estados más intensamente. La energía $\mathrm{E}_{c}$ que separa los estados no localizados de los que sí lo están es el límite de la movilidad. El salto variable $(16,24)$ es el mecanismo por el cual un electrón se traslada por medio de orbitales localizados en un potencial aleatorio, observándose una distribución aleatoria de distancias de salto. Un ejemplo es el caso del $\mathrm{Sr}\left(\mathrm{Ti}_{1-\mathrm{x}} \mathrm{Fe}_{\mathrm{x}} \mathrm{O}_{3}(24)\right.$.

\subsubsection{SUPERCONDUCTIVIDAD}

Es un fenómeno fundamental, que no puede ser tratado en ausencia de la interacción electrón-fonón. La estructura perovskita tiene un importancia por sí misma entre los superconductores y se podría considerar que los materiales cerámicos perovskíticos con esta propiedad forman su propio grupo de superconductores de alta temperatura. Daremos, siquiera someramente, una explicación teórica del proceso restringiéndonos al modelo BCS $(25,1)$. Esta teoría se basa en el hecho de que la reducción en energía asociada al emparejamiento de dos electrones de spines opuestos en presencia de una deformación de la red puede superar a la repulsión coulombiana de dichas cargas. En este caso la interacción neta será atractiva, haciendo inestable el mar de Fermi de los electrones de conducción. Se puede tener así una ligadura vía fonones entre electrones conformando los llamados pares de Cooper que son estados ligados con momentos $\mathrm{K}$ y spines opuestos. El estado fundamental superconductor está formado por todos los pares de Cooper, que por lo tanto, no actúan independientemente. Para romper uno de ellos hay que suministrar energía de tipo térmico (temperatura crítica superconductora), o bien magnético (campo crítico). Por ello, mientras no se suministre una energía superior a ese gap, los electrones forman pares y se mueven con un momento K bien determinado, que no cambia, conduciendo sin resistencia (en una red cristalina es el cambio del $\mathrm{K}$ de los electrones de conducción debido a la interacción con los fonones lo que produce la resistividad). Los únicos electrones que pueden intervenir en este proceso son los que tienen energías próximas al nivel de Fermi. Existen modelos más complejos $(26,27)$ pero la base fundamental es la explicada.

Tanto la superconductividad como la conducción polarónica precisan del estudio del enfrentamiento de la tendencia a la colectividad o al acoplo electrón-fonón (16), pero se diferencian en que, en la primera, existe una atracción entre electrones mediatizado por los fonones que es inmediata mientras que en la segunda tenemos la repulsión coulombiana. Todo se debe a la relación entre $\mathrm{b}$ y la frecuencia $\omega$ del fonón: $b>(\omega$ supone la aparición de un potencial estático medio para los electrones debido a la deformación de la red ya que el fonón no puede seguir al electrón, la consecuencia es la localización de la carga y por tanto la aparición de la conducción polarónica, mientras que si $\omega>b$ los fonones pueden seguir el movimiento de los electrones instantáneamente dando lugar a la formación de los pares de Cooper sin dificultad alguna y por lo tanto al estado superconductor. Como se verá en la segun- 
da parte de este trabajo, existe un valor crítico $\mathrm{b}_{\mathrm{s}}$ que limita la aparición de la superconductividad.

Dentro de la estructura perovskita y de los óxidos cerámicos son los cupratos $\left(\mathrm{LaCuO}_{4}\right.$ (28), $\mathrm{La}_{2} \mathrm{CuO}_{5}$ y $\left.\mathrm{SrCu}_{2} \mathrm{O}_{3}(29)\right)$ los que han despertado mayor interés en este campo con el ya clásico $\mathrm{YBa}_{2} \mathrm{Cu}_{3} \mathrm{O}_{7}$, sin embargo las posibilidades de sustitución del cobre son múltiples $\mathrm{YBa}_{2} \mathrm{Cu}_{3-\mathrm{x}} \mathrm{Fe}_{\mathrm{x}} \mathrm{O}_{7}(30), \mathrm{YBa}_{2}\left(\mathrm{Cu}_{1-\mathrm{y}} \mathrm{Co}_{\mathrm{y}}\right)_{3} \mathrm{O}_{\mathrm{x}}$ (31), $\mathrm{Bi}_{2} \mathrm{Sr}_{2} \mathrm{Ca}\left(\mathrm{Cu}_{1-x} \mathrm{M}_{x}\right) \mathrm{O}_{y}$ con $\mathrm{M}=\mathrm{Fe}$, Co (32). Otras posibles composiciones de interés contienen níquel $\left(\mathrm{La}_{2-x} \mathrm{Sr}_{x} \mathrm{NiO}_{4}(33)\right)$ o bismuto $\left(\mathrm{Ba}_{1-\mathrm{x}} \mathrm{K}_{\mathrm{x}} \mathrm{BiO}_{3}(34), \mathrm{Ba}\left(\mathrm{Pb}_{075} \mathrm{Bi}_{0.25}\right) \mathrm{O}_{3}(35)\right)$.

Es interesante la comparación de los datos obtenidos por Rajeev y Raychaudhuri (36) para las perovsquitas $\mathrm{LaNiO}_{3}$ y $\mathrm{YBa}_{\mathrm{e}} \mathrm{Cu}_{3} \mathrm{O}_{7}$ comprobando que son similares en el caso del estado normal del último, sirviendo el niquelato, por tanto, como modelo para todos los óxidos metálicos habituales y para el estado normal de los que son además superconductores. Un estudio a muy bajas temperaturas de la composición anterior podría mostrar resultados interesantes en este campo.

TABLA II. COMPARACIÓN DE LAS PROPIEDADES ELECTRÓNICAS BÁSICAS DEL $\mathrm{LaNiO}_{3}$ Y EL YBa $\mathrm{Cu}_{3} \mathrm{O}_{7}$ SEGÚN RAJEEV Y RAYCHUDHURI (36).

\begin{tabular}{|l|l|l|}
\hline PROPIEDAD & $\mathbf{L a N i O}_{3}$ & $\mathrm{YBa}_{2} \mathbf{C u}_{3} \mathbf{O}_{7}$ \\
\hline Densidad electrónica $\mathrm{n}\left(\mathrm{cm}^{-3}\right)$ & $1.710^{22}$ & $0.610^{22}$ \\
Temperatura de Debye $(\mathrm{K})$ & 420 & 370 \\
Nivel de Fermi $(\mathrm{eV})$ & 0.21 & 0.12 \\
$\begin{array}{l}\text { Densidad de estados } \mathrm{N}\left(\mathrm{E}_{\mathrm{F}}\right) \\
\left(\mathrm{eV}^{-1} \mathrm{~cm}^{-3}\right)\end{array}$ & $1.1 \times 10^{23}$ & $0.7 \times 10^{23}$ \\
$\begin{array}{l}\text { Masa efectiva } \mathrm{m}_{\mathrm{e}} / \mathrm{m}_{\mathrm{o}}\left(\mathrm{m}_{\mathrm{o}}=9.1\right. \\
\left.10^{-31} \mathrm{~kg}\right)\end{array}$ & 11 & 9.6 \\
$\mathrm{~A}$ la temperatura de $273 \mathrm{~K}:$ & $3.410^{-3}$ & $3.710^{-3}$ \\
\hline$(1 / \rho)(\mathrm{d} \rho / \mathrm{dT})\left(\mathrm{K}^{-1}\right)$ & \\
\hline
\end{tabular}

\subsection{Transiciones metal-aislante.}

Como hemos visto hasta aquí, existe un amplio margen de comportamientos conductores en la estructura que estudiamos, y pueden aparecer transiciones entre ellos. Estas transformaciones dan lugar a discontinuidades espectaculares en la conductividad frente a variaciones de parámetros como la temperatura, la presión o la concentración de impurezas. Seguiremos aquí la clasificación que Adler(19) hace de estos procesos.

\subsubsection{TRANSICIONES RELACIONADAS CON EL SOLAPAMIENTO DE BANDAS.}

Serán inducidas por presión o temperatura. El proceso implica una modificación del gap energético con las magnitudes citadas hasta un valor crítico en el cual se anula y comienza el solapamiento entre bandas, la transición no puede ser gradual sino brusca. Los modelos son:

(1) Transición termodinámica de primer orden: se da un repentino cambio de signo de la energía del gap cuando se alcanza el valor crítico del parámetro modificable (presión y volumen de la celda primitiva que se relaciona con la estruc- tura electrónica, o temperatura y densidad de estados en conexión con los cambios entrópicos).

(2) Cambios en la estructura cristalina: Una transformación de la fase cristalográfica que implique un cambio en la densidad electrónica de estados y genere un solapamiento de las bandas.

(3) Transición a un aislante excitónico $(14,22)$ : Introducimos aquí la interacción coulombiana entre los electrones y los huecos que da lugar a la formación de excitones: $\mathrm{V}=-\mathrm{e}^{2} / \mathrm{e}_{0} \mathrm{r}$, donde $r$ es la distancia entre las dos cargas. En el caso de un metal con intenso solapamiento entre bandas esta transición no es probable porque la alta concentración de carga libre apantalla esta fuerza, en cambio en un semimetal de pequeño solapamiento inicial esta interacción puede dar lugar a la formación de un gap y un semiconductor de banda estrecha puede transformarse en un aislante excitónico.

El material reduce su energía formando excitones entre electrones por debajo de $\mathrm{E}_{\mathrm{F}} \mathrm{y}$ huecos por encima siempre que la energía de enlace sea mayor que el gap en el semiconductor o que el solapamiento en el semimetal. El proceso será espontáneo mientras la energía de formación de nuevos pares electrón hueco (función de la densidad de excitones ya presentes) sea menor que la de enlace del excitón. Si las cargas disponibles conforman mayoritariamente excitones el proceso de conducción se reduce notablemente por su carácter neutro y estamos en presencia de un aislante excitónico. Se trata de un ejemplo de condensación de Bose y por ello sólo es estable por debajo de cierta temperatura crítica. Este estado viene acompañado de una reducción en la simetría del cristal que se logra a través de un ordenamiento antiferromagnético o bien de una deformación de la red .

\subsubsection{TRANSICIONES INDUCIDAS ELECTRONICAMENTE.}

Existen dos tipos de transiciones que se pueden incluir en esta sección:

(1) Deformaciones cristalinas: esta clase de transiciones se basa fundamentalmente en que en un material de banda estrecha, a bajas temperaturas, el estado metálico puede ser inestable ante deformaciones cristalinas que disminuyan la simetría. El caso más simple para nuestro interés es el de un óxido en que los iones metálicos se desplazan de sus posiciones de equilibrio, modifican la periodicidad y el tamaño de las zonas de Brillouin, y como consecuencia aparece un gap. En el caso de conducción metálica y a bajas temperaturas puede suceder que el efecto de la deformación logre que los estados ocupados estén solamente por debajo del gap, surgiendo la transición a un aislante o semiconductor. Otra posibilidad es que se separe una banda enlazante de otra antienlazante por el gap que decrece linealmente con la concentración de carga libre. Esta situación puede llevar a una transición semiconductor-metal a cierta temperatura crítica que dependerá del valor del gap inicial y de la deformación relativa. Sólo en el caso de materiales de banda estrecha tiene lugar una deformación espontánea a bajas temperaturas ya que tanto la energía de los estados electrónicos ocupados como la de la red debe reducirse.

(2) Ordenamiento magnético: Slater(14) señala que el orden antiferromagnético puede considerarse, en lo referido a los efectos sobre la estructura electrónica de bandas, equivalente a la deformación previamente estudiada al producir el desdoblamiento de lo que inicialmente era una única banda, en otras dos con electrones de spines opuestos cuya diferencia en 
energías da lugar a un gap. Por encima de la tempertaura de Néel desaparece el orden magnético y por tanto existe una banda única, dicha banda está semillena, y puede asegurar que el estado para $\mathrm{T}<\mathrm{T}_{\mathrm{N}}$ es semiconductor, $\mathrm{y}$ por lo tanto surge una transición metal semiconductor a esa $\mathrm{T}_{\mathrm{N}}$.

\subsubsection{TRANSICIONES DE MOTT.}

Los aislantes de Mott $(14,16,20)$ son materiales de los que podría esperarse un comportamiento de tipo banda estrecha pero cuyos electrones de conducción permanecen localizados en los átomos. La relación entre la anchura de banda (a través de b o D) y la repulsión coulombiana U nos permite dilucidar cuando se da esa situación:

anchura de banda/U $>>1$-formación de la banda, $<<1$-localización de Mott

La transición entre un estado aislante de Mott y un metal sucederá para un valor crítico de esa magnitud que puede ser modificada por los siguientes paràmetros:

(1) Concentración de carga libre: Cuando es baja en un aislante de Mott se forman excitones no conductores, cuando es alta las interacciones coulombianas se apantallan. Para un cierto valor crítico de $\mathrm{n}$ el apantallamiento es tal que ya no aparecen excitones, generando, de forma repentina, un gran número de cargas que participan en la conducción transformando el aislante de Mott en un metal .

(2) Presión: al aumentar la presión el espaciado interatómi$\mathrm{co}$, a, decrece y por lo tanto el solapamiento entre orbitales crece ensanchando la banda. Existe un valor crítico para el cual se produce la transición aislante de Mott-metal.

(3)Temperatura: A través de la excitación térmica puede surgir una concentración de carga libre importante que apantalle las interacciones coulombianas reduciendo $\mathrm{U}$ dando lugar a la transición.

(4)Radio iónico de A: Este parámetro modifica el ángulo de enlace B-O-B de modo que genera un cambio en la energía de transferencia y como consecuencia en la anchura de las bandas y del gap del semiconductor. Se observa así una dependencia de estas transiciones de Mott en relación con la anchura de las bandas del semiconductor de tipo perovskita. Es el caso de las cobaltatos (37)

En general resulta muy dificil distinguir el origen de una transición metal-aislante ya que pueden combinarse varios procesos, o que los efectos asociados a la de reducción de la simetría pueden encubrir el verdadero detonante de la situación.

\section{CONCLUSIONES.}

Se ha hecho una revisión de los distintos modelos teóricos propuestos en la literatura y de las propiedades de transporte electrónico que están presentes en los óxidos cerámicos tipo perovskita citados, en conexión con la estructura de bandas. La predicción, a partir de dichos modelos, o el diseño de las propiedades de los materiales deseados en función de la composición es tentador y no deja de ser uno de los principales objetivos de la Ciencia del Estado Sólido. El presente trabajo y su continuación no son sino un intento de relacionar las propuestas teóricas desarrolladas hasta el momento de modo que pueda tenerse una visión global e integradora las propiedades físicas de estos compuestos y su comprensión en base a parámetros controlables y previsibles.
TABLA III. PropiedAdES DE ALGUNOS ÓXIDOS CON METALES DE TRANSICIÓN 3D Y ESTRUCTURA PEROVSKITA. ELABORACIÓN PROPIA DATOS RECOGIDOS DE GoOdenOUgh ( 2) Y DE Muller (9).

\begin{tabular}{|c|c|c|c|c|c|}
\hline Compuesto & Simetría & \begin{tabular}{|l|} 
Configuración \\
propuesta para \\
los electrones d
\end{tabular} & \begin{tabular}{|l|} 
Valor total del \\
spin en el catión \\
B en el límite de \\
electrones \\
localizados \\
\end{tabular} & \begin{tabular}{|l|} 
Tipo de \\
conductividad \\
(d $\sigma / \mathrm{dT}>0$ semic, \\
$<0$ metal)
\end{tabular} & \begin{tabular}{|l|} 
Integrales de \\
solapamiento
\end{tabular} \\
\hline $\mathrm{SrFeO}_{3}$ & Cúbica & $\mathrm{t}_{\alpha}{ }^{* 3} \pi_{\beta}{ }^{* 1} \sigma^{* 0}$ & 1 & metálica & $\begin{array}{l}\Delta_{\text {cac }}{ }^{\pi} \\
\leq \Delta_{\text {crit }}<\Delta_{\text {cac }}{ }^{\sigma}\end{array}$ \\
\hline $\mathrm{LaFeO}_{3}$ & Ortorrómbica & $t^{33} e^{42}$ & $5 / 2$ & semiconductora & $\begin{array}{l}\Delta_{\text {cac }}{ }^{\pi} \\
<\Delta_{\text {cac }}{ }^{\sigma}<\Delta_{\text {crit }}\end{array}$ \\
\hline $\mathrm{LaCoO}_{3}$ & $\begin{array}{l}\mathrm{R} 3 \mathrm{c} \leftrightarrow \mathrm{R} 3 \leftrightarrow \mathrm{R} \\
3 \mathrm{~m}\end{array}$ & $\begin{array}{l}\mathrm{t}^{* 6} \sigma^{* 0} \mathrm{a} \mathrm{T}=0^{\circ} \mathrm{K} \\
\mathrm{t}^{* 4} \mathrm{e}^{* 2} \mathrm{si} \\
800^{\circ} \mathrm{K}<\mathrm{T}<121 \\
0^{\circ} \mathrm{K} \\
\text { (es decir, R3) }\end{array}$ & 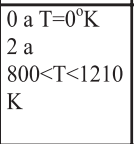 & $\begin{array}{l}\text { semiconduct } \\
\text { or } \Leftrightarrow \text { metal } \\
\left(T_{\text {trans }}=1210^{\circ}\right. \\
\mathrm{K})\end{array}$ & $\begin{array}{l}\Delta_{\mathrm{cac}}{ }^{\pi} \\
<\Delta_{\mathrm{cac}}{ }^{\sigma} \cong \Delta_{\text {crit }}\end{array}$ \\
\hline $\mathrm{LaNiO}_{3}$ & Romboédrica & $t^{* 6} \sigma^{* 1}$ & $1 / 2$ & metálica & $\begin{array}{l}\Delta_{\mathrm{cac}}{ }^{\pi} \\
<\Delta_{\mathrm{crit}}<\Delta_{\mathrm{cac}}{ }^{\sigma}\end{array}$ \\
\hline $\mathrm{CaMnO}_{3}$ & Cúbica & $\mathrm{t}^{* 3} \sigma^{* 0}$ & $3 / 2$ & semiconductora & $\begin{array}{l}\Delta_{\text {crit }}<\Delta_{\mathrm{cac}}{ }^{\pi} \\
<\Delta_{\mathrm{cac}} \sigma\end{array}$ \\
\hline $\mathrm{LaMnO}_{3}$ & $\begin{array}{l}\text { Ortorrómbica } \\
\text { con distorsión } \\
\text { de Janh Teller } \\
(\mathrm{c} / \sqrt{2}<\mathrm{a}<\mathrm{b}) \leftrightarrow \\
\text { Romboédrica }\end{array}$ & $t^{33} e^{* T}$ & 2 & \begin{tabular}{|l|} 
semiconductora \\
(con una \\
disminución \\
en el gap en \\
la transición \\
cristalina a \\
$752^{\circ} \mathrm{K}$ )
\end{tabular} & $\begin{array}{l}\Delta_{\text {cac }}{ }^{\pi} \\
<\Delta_{\text {cac }}{ }^{\sigma}<\Delta_{\text {crit }}\end{array}$ \\
\hline
\end{tabular}

\section{BIBLIOGRAFÍA}

1. N.W.Ashcroft, N.D. Mermin, "Solid State Physics", CBS publishing Asia LTD., 1988.

2. J.B.Goodenough. "Metallic Oxides", Progress in Solid State Chemistry vol 5, Ed. H. Reiss. Pergamon Press, NY 1971, 145-399.

3. C. Kittel "Quantum Theory of Solids", John Willey, N.Y.1987.

4. B.H.Bransden, C.J.Joachain "Physics of atoms and molecules", Harlow, Longman Scientific and technical, 1990.

5. J.B.Goodenough, J.M.Longo "Crystallograpihe and magnetic properties of perovskite and perovskite related compounds" Landolt Börnstein New Series, K.H.Hellwege, A.M. Hellwege, vol. 4, part a, 126. Springer-Verlag, Berlin, 1970.

6. K.W.H.Stevens, “Spin Hamiltonians”, Ed.G.T.Rado, H. Suhl, “Magnetism”, vol 1, 1, 1969

7. J.B.Goodenough "Covalency Criterion for Localized vs Collective Electrons in Oxides with Perovskite Structure",J.Appl.Phys, vol 37, 3, pp 1415-1422, 1966.

8. J.B.Goodenough, P. M. Raccah “Complex vs Band Formation in Perovskite Oxides", J.Appl.Phys, vol 36, 3, pp 1031-1033, 1965.

9. O.Muller, R.Roy "The Mayor Ternary Structural Families", Crystal Chemistry of Non Metallic Materials, Springer-Verlag, 1974.

10. V.M. Goldschmidt "Geochemische Verteilungsgesetze der Elemente", VII, VIII, 1927-28.

11. D.M. Smyth "Defects and order in perovskite related oxides", Ann.Rev.Mater.Sci. 15, 329-57, 1985

12. J.B.Goodenough "Characterization of d electrons in solids by structure II.Spontaneus crystallographic Distortions",Mat.Res.Bull. vol2, 2,165-185, 1967.

13. J.B.Goodenough "Magnetism and Crystal structure in Nonmetals", Ed.G.T.Rado, H. Suhl, “Magnetism”, vol 2, 1, 1969.

14. 14. R.W.Vest, J.M.Honig "Highly Conducting ceramics and the conductor insulator transition", Electrical conductivity in Ceramics and Glass part B, Edt. N. M. Tallan, 1974.

15. 15. H.L.Tuller "Highly conductive ceramics", Ceramic Materials for Electronics, Edt Buchanan, Marcel Dekker, Inc, NY, 1986.

16. N. Tsuda, K. Nasu, A. Yanase, K. Siratori, "Electronic Conduction in Oxides", Springer Series in Solid State Sciences, springer Verlag, Berlin, 1991.

17. J.Nowotny, C.C.Sorrell "Electrical Properties of Oxide Materials", Trans Tech publications, Switzerland ,1997.

18. J.M. Ziman "Electrons and phonons. The Theory of Transport Phenomena in Solids", Oxford Univ. Press, London 1960.

19. H. Frölich, "Elecrons in lattice fields", Adv. Phys.3, 325-361, 1954.

20. D. Adler "The imperfect Solid. Transport Properties", Treatise on Solid State Chemistry, vol. 2, pp 237-332, Ed N.B. Hannay, Plenum Press, NY, 1975. 
21. T. Holstein, "Studies of polaron motion:II. The Small polaron", Ann. Phys. NY, 8, 353-389, 1959.

22. A.J. Bosman, H.J. van Daal, "Small polaron vs Band Conduction in some transition metal oxides", Adv. Phys., vol19, 77, 1970.

23. N.F. Mott: Philos. Mag. 19, 835, 1969.

24. C. Ang, J.R. Jurado, Z. Yu, M.T. Colomer, J.R. Frade, J.L. Baptista, "Variable range hopping conduction and dielectric relaxation in disordered $\mathrm{Sr}_{0.97}\left(\mathrm{Ti}_{1}\right.$ $\left.{ }_{x} \mathrm{Fe}_{\mathrm{x}}\right) \mathrm{O}_{3-\mathrm{y}}$ ", Phys. Rev. B, vol 57, 19, 1998

25. J. L. Vicent "Superconductividad", Física Cuántica (II), C. Del Río, Eudema, Madrid 1991.

26. H. Roder, H. Feshke, R.N. Silver, “ The ordering of polarons in the Holstein t-J model, an application to $\mathrm{La} \mathrm{Sr} \mathrm{NiO}$ ", Europhys. Lett. , vol 8, 4, 1994

27. O. Narikiyo, K. Miyake, "Itinerant localized duality picture of spin fluctuation in high $\mathrm{T}_{\mathrm{c}}$ cuprates II. A unified phenomenology", J. Phys Soc Jpn, vol $63,11,1994$.

28. A. Daridon et al, "Growth and electrochemical oxidationes of MBE grown c-axis $\mathrm{LaCuO}_{4}$ thin films on different sustrates", J Alloys Compd, vol 251, 12, 1997.

29. P. Germain, M. Lagues, "Electron structure of the copper oxidesladder compounds $\mathrm{La}_{2} \mathrm{Cu}_{2} \mathrm{O}_{5}$ and $\mathrm{SrCu}_{2} \mathrm{O}_{3}$ in a weak coupling model", J Alloys Compd, vol 251, 1-2, 1997 .
30. V.F. Masterov et al, "Superconductivity and magnetic order in the copper sublattices of the $\mathrm{Yba}_{\mathrm{e}} \mathrm{Cu}_{3-\mathrm{x}} \mathrm{Fe}_{\mathrm{x}} \mathrm{O}_{7+\mathrm{y}}$ ceramics", JETP Lett, vol 60, 6, 1994.

31. W. Wu et al, "Neutron and XRD studies on $\mathrm{YBa}_{2}\left(\mathrm{Cu}_{1-\mathrm{y}} \mathrm{Co}_{\mathrm{y}}\right) \mathrm{O}_{\mathrm{x}}$ superconductors with $0.02<y<0.04$ ", Physuca C, vol 282-287, 1998.

32. V.E.Gasumyants et al, "Effect of substitution for $\mathrm{Cu}$ in $\mathrm{Bi}_{2} \mathrm{Sr}_{\mathrm{s}} \mathrm{Ca}\left(\mathrm{Cu}_{1-\mathrm{x}} \mathrm{M}_{\mathrm{x}}\right) \mathrm{O}_{\mathrm{y}}$ $(\mathrm{M}=\mathrm{Fe}, \mathrm{Co})$ on thermopower and band spectrum in the normal state", Physica C, vol 282-287, 1997.

33. T. Mizokawa, A. Fujimori, "Hartree Fock sudy of charge ordered states in $\mathrm{La}_{2,2} \mathrm{Sr}_{x} \mathrm{CuO}_{4}$ and $\mathrm{La}_{2-\gamma} \mathrm{Sr}_{x} \mathrm{NiO}_{4}$ ", Phys Rev B, vol 56, 18, 1997.

34. M. E. Kozlov, "Change of IR spectra of $\mathrm{Ba}_{1-\mathrm{x}} \mathrm{K}_{\mathrm{x}} \mathrm{BiO}_{3}$ crystals at the low tempertaure phase transition", Phys Rev B, vol 56, 19, 1997.

35. Z. L. Miskovic, " Phono dispersion in $\mathrm{Ba}\left(\mathrm{Pb}_{0.75} \mathrm{Bi}_{0.25}\right) \mathrm{O}_{3}$ ", Phys Rev A, vol 57, 1, 1998.

36. K. P. Rajeev, A. K. Raychaudhuri, "Quantum corrections to the conductivity in a perovskite oxide: Alow temperature study of $\mathrm{LaNi}_{1-x} \mathrm{Co}_{\mathrm{x}} \mathrm{O}_{3}(0<\mathrm{x}<0.75)^{\prime \prime}$, Phys Rev, B, vol 46, 3, 1992.

37. S.Yamaguchi, Y. Okimoto, Y.Tokura, "Bandwith dependence of insulatormetal transitions in perovskite cobalt oxides", Phys.Rev.B, vol 54, 15, 1996.

\title{
CHInf ares 2001 9TH CHINA INTERNATIONAL GLASS INDUSTRIAL TECHNICAL EXHIBITION
}

\author{
Being Exhibition Center (newly renovated) \\ April 2002
}

Organizer: The Chinese Ceramic Society

Co-organizer: China National Assotiation for Glass Industry

- the largest international Glass Exhibition in Asia - One of the Most Influential International Glass Exhibitions in the World

\begin{abstract}
This exhibition is the only international glass exhibition in China sponsored and supported by all glass-related government departments and relevant trade associations. It has become the largest one in Asia for the previous four events since 1994 and the number of exhibitors is over 300 and the exhibition are reaches 15.000 square
\end{abstract} meters. It is one of the most influential international glass exhibitions in the world.

\$ SCALE OF this GLASS EXHibition IS EXPECTED to be MUCH LARGer than PREVIOUS ONES

MAJOR SCOPE EXHIBITION 品

\section{Glass Products.}

(1) Architectural glass and decoration glass:

(2) Fenestration and hardware;

(3) Industrial glass and automotive glass

(4) Glassware: table glass and automotive glass;

(5) Glass containers: drinking, wine, medical and exquisite cosmetic bottles;

(6) Electronic glass and electric lighting glass;

(7) Glass fiber and glass wool;

(8) Special glass: silica glass, art glass, glass-ceramics, etc.

II. Instrument, Equipment and Automatic Control System.

(1) Weighing, dosing, mixing and refining equipment for raw and mixed materials;

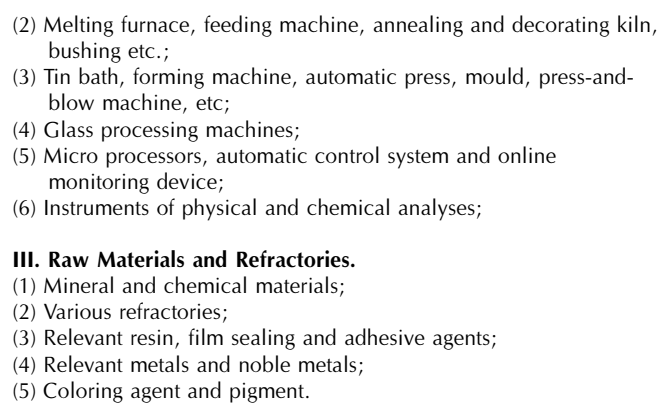

III. Raw Materials and Refractories.

1) Minerat and chemical materials;

(2) Various refractories;

(3) Relevant resin, film sealing and adhesive agents;

(4) Relevant metals and noble metals;

(5) Coloring agent and pigment. 